\title{
Lift movement detection with QDA classifier for an active hip exoskeleton
}

\author{
Baojun Chen, Lorenzo Grazi, Francesco Lanotte, Nicola Vitiello, Simona Crea
}

\begin{abstract}
In the recent years, the interest in exoskeletons for industrial applications has significantly increased. To provide efficient assistance to users with an active exoskeleton when performing lifting tasks, the automatic detection of the movement onset in real time is an important requirement for the control system. In this paper, we propose a lift detection algorithm using only exoskeleton sensors. A quadratic discriminant analysis classifier is combined to threshold-based rules to detect the onset of the lift movement. The promise of this algorithm was validated by applying it to control online an active hip exoskeleton in a sequence of experimental tasks including lifting and lowering a load, standing up, sitting down, and walking. The average detection accuracy over seven healthy subjects was $98.72 \pm 0.58 \%$.
\end{abstract}

\section{INTRODUCTION}

$\mathbf{I}^{\mathrm{N}}$ $\mathrm{N}$ recent years, the interest in exoskeletons for industrial applications has continuously and significantly increased [1]. Exoskeletons have the potential to relieve part of the effort workers have to sustain during their job, especially in heavy material handling tasks. Compared to passive exoskeletons, active exoskeletons have more versatile control systems and thanks to the external power source they can produce more powerful and more efficient lift assistance. However, to take full advantages of an active exoskeleton, it is important for the control system to automatically detect the onset of the lift movement in order to deliver appropriate assistance synchronously with the assisted movement.

Despite the necessity of developing lift detection algorithms for active exoskeletons, related studies are limited $[2,3]$. Kawai et al. developed a myoelectric controller to detect user's lift intentions to trigger lift assistance [2]. Electromyographic (EMG) signals of three thigh muscles were collected and processed with artificial neural network (ANN). The main limitation of this approach is that extra EMG electrodes should be placed on user's body, which makes it inconvenient to use. In addition, the performance of lift detection might be influenced by EMG changes due to muscle fatigue, sweating, and electrode displacement, which could happen after performing lifting tasks for a long time.

This work was supported by Regione Toscana within the CENTAURO project (Bando FAR-FAS 2014) and EU within the HUMAN project (H2020-FOF-2016, GA 723737).

Baojun Chen, Lorenzo Grazi, Francesco Lanotte, Simona Crea are with The BioRobotics Institute, Scuola Superiore Sant'Anna, Pisa, Italy.

Nicola Vitiello is with the BioRobotics Institute, Scuola Superiore Sant'Anna, Pisa, Italy and Fondazione Don Carlo Gnocchi, Firenze, Italy.

Baojun Chen is corresponding author to provide email: baojun.chen@santannapisa.it.
In this paper, we present a lift detection algorithm using only signals collected by exoskeleton sensors. Compared to algorithms requiring extra sensors (e.g. EMG electrodes), our approach is more practical to be used in real working scenarios. The algorithm first detects possible lift movements, and then a quadratic discriminant analysis (QDA) classifier is used to determine whether they are real lifts or other movements. The algorithm was verified on seven healthy subjects wearing an active hip exoskeleton.

\section{METHODS}

\section{A. Experimental setup and protocol}

The exoskeleton used in this study is a robotic hip exoskeleton to assist the hip flexion/extension movement (APO [4]), developed at The BioRobotics Institute of Scuola Superiore Sant'Anna. Sensors used for lift detection include two on-board encoders and an inertial measurement unit (IMU) on the backpack (Fig. 1(A)). They are used to record hip joint angles of both sides and kinematic information of the trunk. A $5-\mathrm{kg}$ box is used in the experiment.

Seven healthy male subjects $(27.9 \pm 2.3$ years old, $178.1 \pm$ $8.1 \mathrm{~cm}, 70.0 \pm 6.4 \mathrm{~kg}$ ) were recruited for this experiment. The experiment consisted of two sessions: training session and testing session. In the training session, subjects were asked to perform two types of tasks: (1) repetitive lifting and (2) a movement sequence including standing up, walking, lifting and lowering the load, and sitting down. Both tasks were performed with five different lift techniques (namely, squat, stoop, freestyle, left-asymmetric, and right-asymmetric lifting). In the testing session, subjects were asked to perform the task similar to the second task in the training session with only freestyle lift technique at slow, normal and fast speeds.
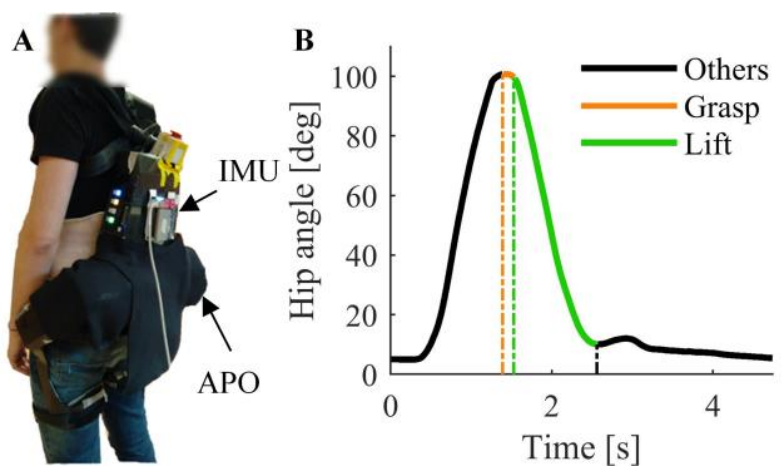

Fig. 1. (A) A subject wearing the APO endorsed with an IMU on the backpack. (B) Phase definition for lift detection. The dotted lines represent in the order the start of the Grasp phase, the start of the Lift phase and the end of the Lift phase. 
The APO was controlled in transparent mode (i.e. zero-torque control) in the training session, while in assistive mode (assistance delivery was triggered by the lift detection algorithm) in the testing session. Data collected in the training session were used to train the detection model used in the testing session.

\section{B. Lift detection algorithm}

Lift detection is performed in two steps. In the first step, a set of threshold-based rules is used to detect possible lift movements. Subject's movement can be segmented into three phases: Grasp, Lift, and Other (Fig. 1(B)). The transition from Other to Grasp should follows three rules: (1) $\theta_{\text {diff }}<$ $\alpha_{1}$, (2) $\theta_{\text {mean }}>\alpha_{2}$ and (3) $\theta_{\text {std }}<\alpha_{3}$. The transition from Grasp to Lift, i.e. the detection of possible lift, should satisfy three rules: (1) $\theta_{\text {std }}>\alpha_{4}$, (2) HasPeak $=1$, and (3) $T_{\text {grasp }} \leq T_{0}$. The transition from Lift to Other should meet two rules: (1) $\theta_{\text {mean }}<\alpha_{5}$, and (2) $\theta_{\text {std }}<\alpha_{6}$ or HasValley $=1$. In the above rules: $\theta_{\text {diff }}$ is calculated as $\left|\theta_{L}-\theta_{R}\right|$, where $\theta_{L}$ and $\theta_{R}$ denote hip joint angle of the left and right side, respectively; $\theta_{\text {mean }}$ equals to $\left(\theta_{L}+\theta_{R}\right) / 2$ and $\theta_{\text {std }}$ is the standard deviation of $\left(\theta_{L}+\theta_{R}\right)$ over the last 100 $\mathrm{ms}$; $\mathrm{T}_{\text {grasp }}$ is the current duration of grasping; HasPeak equals to 1 if a peak of $\left(\theta_{L}+\theta_{R}\right)$ has occurred within the Grasp phase, otherwise it equals to 0; Has Valley equals to 1 if a valley of $\left(\theta_{L}+\theta_{R}\right)$ is detected within the Lift phase, otherwise it equals to $0 ; \alpha_{1}$ to $\alpha_{6}$, and $T_{0}$ are predefined thresholds.

In the second step, if a possible lift movement is detected, a QDA classifier is used to determine whether it is a real one or not. Two features are used for the recognition, which are $f_{1}=$ $\theta_{\text {mean }}$ and $f_{2}=\beta(i)-\beta\left(i_{\text {grasp } 0}\right) . \quad \beta$ is defined as $\theta_{\text {mean }}-\varphi$, where $\varphi$ is roll value of the IMU on the backpack of the exoskeleton. $i$ denotes the current sample and $i_{\text {graspo }}$ denotes the sample at the initial moment of grasping in current lift.

\section{RESULTS}

Table. I reports the performance of online lift detection in the testing session. Note that TP denotes lift correctly detected as lift; FP denotes non-lift movement mistakenly detected as lift; TN denotes non-lift movement not detected as lift; FN denotes lift not detected as lift.

The average detection accuracy achieved over seven subjects is $98.72 \pm 0.58 \%$ (Mean \pm SEM).

TABLE I

LIFT DETECTION PERFORMANCE IN THE TESTING SESSION

\begin{tabular}{cccccc}
\hline \hline Subject & TP [\#] & FP [\#] & TN [\#] & FN [\#] & Accuracy \\
\hline S 1 & 174 & 0 & 29 & 0 & $100.00 \%$ \\
S 2 & 177 & 0 & 30 & 3 & $98.57 \%$ \\
S 3 & 183 & 8 & 22 & 1 & $95.79 \%$ \\
S 4 & 180 & 1 & 29 & 0 & $99.52 \%$ \\
S 5 & 182 & 1 & 29 & 0 & $99.53 \%$ \\
S 6 & 180 & 5 & 25 & 0 & $97.62 \%$ \\
S 7 & 180 & 0 & 30 & 0 & $100.00 \%$ \\
\hline \hline
\end{tabular}

\section{DISCUSSION AND CONCLUSION}

To better provide assistance to a user, movement intention detection plays an important role in active exoskeletons control. In this paper, we develop a lift detection algorithm for an active hip exoskeleton aiming at lift assistance. The presented algorithm uses a two-step detection strategy. The first step could detect the onset of a possible lift movement and avoid most mistaken detection of non-lift movements (e.g. standing up and level-ground walking) as lift. However, some movements (e.g. sitting down) could be sometime mistakenly detected as lift: indeed, most of the errors are due to the misclassification of sitting down as lift. Therefore, in the second step, a QDA classifier is necessary to further verify whether the detected lift is actually occurring.

The proposed algorithm has two main advantages. First, the algorithm only uses signals measured from exoskeleton embedded sensors, which makes the whole system compact and convenient to use, especially for industrial application. Second, the algorithm is able to detect the lift onset timely enough to provide efficient assistance. Subjective feedback from all the subjects showed that the APO controlled by the proposed algorithm could provide helpful assistance in the testing session, which made the lift easier with less perceived physical effort. In addition, the application of a QDA classifier in the second step increases the potential of the algorithm with respect to a rule-based strategy, as we did in [4], because QDA classifier can be used to solve a multi-class recognition problem, as well. For example, in addition to detecting the lift, it might be possible for the algorithm to classify the lift technique.

The primary limitation of the algorithm is that mistaken lift detections could still happen and there are also a few miss detections of lifts. In future works, we will investigate some other features to improve the performance of lift detection, and we will also explore the potential of this algorithm to recognize different lift techniques.

\section{REFERENCES}

M. P. de Looze, T. Bosch, F. Krause, K. S. Stadler, and L. W. O'Sullivan, "Exoskeletons for industrial application and their potential effects on physical work load," Ergonomics, vol. 59, pp. 671-681, 2016.

[2] S. Kawai, H. Yokoi, K. Naruse, and Y. Kakazu, "Study for control of a power assist device. Development of an EMG based controller considering a human model," in 2004 IEEE/RSJ International Conference on Intelligent Robots and Systems, 2004, pp. 2283-2288.

[3] K. Naruse, S. Kawai, H. Yokoi, and Y. Kakazu, "Development of wearable exoskeleton power assist system for lower back support," in 2003 IEEE/RSJ International Conference on Intelligent Robots and Systems, 2003, pp. 3630-3635.

[4] B. Chen, L. Grazi, F. Lanotte, N. Vitiello, and S. Crea, "A Real-Time Lift Detection Strategy for a Hip Exoskeleton," Frontiers in Neurorobotics, vol. 12, p. 17, 2018. 